\title{
Early executive function deficit in preterm children and its association with neurodevelopmental disorders in childhood: a literature review
}

\author{
Jing Sun ${ }^{1, *}$ and Nicholas Buys ${ }^{2}$ \\ ${ }^{1}$ School of Public Health and Griffith Health Institute, \\ Griffith University, Logan Campus, Meadowbrook, \\ Queensland, Australia \\ ${ }^{2}$ Health Group and Griffith Health Institute, Griffith \\ University, Gold Coast Campus, Parkland, Queensland, \\ Australia
}

\begin{abstract}
The purpose of this study is to examine the association of deficits of executive function (EF) and neurodevelopmental disorders in preterm children and the potential of assessing $\mathrm{EF}$ in infants as means of early identification. EF refers to a collection of related but somewhat discrete abilities, the main ones being working memory, inhibition, and planning. There is a general consensus that EF governs goal-directed behavior that requires holding those plans or programs on-line until executed, inhibiting irrelevant action and planning a sequence of actions. EF plays an essential role in cognitive development and is vital to individual social and intellectual success. Most researchers believe in the coordination and integrate cognitive-perceptual processes in relation to time and space, thus regulating higher-order cognitive processes, such as problem solving, reasoning, logical and flexible thinking, and decision-making. The importance of the maturation of the frontal lobe, particularly the prefrontal cortex, to the development of EF in childhood has been emphasized. Therefore, any abnormal development in the prefrontal lobes of infants and children could be expected to result in significant deficits in cognitive functioning. As this is a late-maturing part of the brain, various neurodevelopmental disorders, such as autism spectrum disorders, attention deficit hyperactivity disorder, language disorders, and schizophrenia, as well as acquired disorders of the right brain (and traumatic brain injury) impair EF, and the prefrontal cortex may be particularly susceptible to delayed development in these populations. The deficits of EF in infants are persistent into childhood and related to neurodevelopmental disorders in childhood and adolescence.
\end{abstract}

*Corresponding author: Jing Sun, PhD, School of Public Health, Griffith University and Griffith Health Institute, Griffith University, Logan Campus, Meadowbrook, Queensland 4131, Australia E-mail: j.sun@ griffith.edu.au

Submitted September 3, 2011. Revised October 5, 2011. Accepted October 16, 2011. Previously published online January 13, 2012.
Keywords: developmental disorders; executive function; full-term infants; preterm infants.

\section{Introduction}

There is an increasing incidence of preterm delivery, and the survival rate of preterm newborns is rising because of the advanced assisted reproductive technology and the improvement of technology in obstetrics and in neonatology. The survival rate in preterm birth is increasing with progressive increasing gestational ages (1) because of the improvement of neonatal intensive care. The survival rate of infants born at 25-25 weeks of gestation was $88 \%-100 \%$ in 2010 (2) compared with 36\%-47\% in 1994 (3). In preterm newborns, prenatal, perinatal, and postnatal factors can give rise to adverse neurological outcomes through complex causal pathways (4). The main factors are brain injury (i.e., white matter damage, intraventricular hemorrhage, periventricular hemorrhage, and cortical and deep gray matter damage), and subsequent adverse clinical outcomes and increased incidence of disabilities increase with decreasing gestational age (5). As a significant proportion of brain growth, development, and networking occur approximately during the last 6 weeks of gestation (6), any injury or medical complications in the brain during this time can lead to adverse neurodevelopmental outcomes. The spectrum of neurodevelopmental disorders in preterm children is wide and is represented by cerebral palsy (CP), developmental coordination disorder, neurosensorial impairment, including peripheral and/or central hearing and visual impairments, cognitive impairment, learning disabilities, and psychiatric disorders [i.e. attention deficit hyperactivity disorder, conduct problems, and emotional symptoms (7)]. These disorders have been found to be related to executive function (EF) deficits $(4,8)$.

EF refers to a collection of high-order cognitive abilities, the main ones being working memory, inhibition, planning, flexibility, and shift attention $(9,10)$. There is a general consensus that EF governs goal-directed behavior that requires holding those plans or programs on-line until executed, inhibiting irrelevant action, planning a sequence of actions, attention to distraction, being able to flexibly adjust to changed circumstances or new information (9). EF plays an essential role in cognitive development and is vital to the individual's social and intellectual success. It is thought by most researchers to coordinate and integrate cognitive-perceptual processes in relation to time and space, thus regulating higher-order EFs, such as problem solving, reasoning, logical and flexible thinking, and decision-making. 
The importance of the maturation of the frontal lobe, and in particular, the prefrontal cortex, to the development of EF in childhood has been emphasized by several researchers. Given the important role of EF in cognitive development, any abnormal development in the prefrontal lobes of infants and children could be expected to result in significant deficits in cognitive functioning $(11,12)$. As this is a late-maturing part of the brain, various neurodevelopmental disorders, such as autism spectrum disorders, attention deficit hyperactivity disorder, developmental language disorders, and schizophrenia, as well as acquired disorders of the right brain (and traumatic brain injury) impair EF, and the prefrontal cortex may be particularly susceptible to delayed development in these populations. The current review aims the following:

1. Examine the relationship between preterm birth and the development of prefrontal cortex and EF deficits;

2. Describe EF deficits in infants that persist into childhood and are related to neurodevelopmental disorders in childhood;

3. Explore possible assessment approaches to infant EF through early identification of EF deficits.

\section{The development of the prefrontal cortex in infants}

Early birth has an influence on brain development and timing of neurobiological processes (13). These processes include neuronal migration and differentiation, axon and dendrite sprouting, synapse formation, myelination, programmed cell death, and the persistence of transient structures (i.e., the subplate). Compared with animals, the central nervous system of humans is late to reach full maturity when measured in terms of the number and volume of cells and the size and number of the dendritic spines of its neurons (14). Brain development begins early and advances quickly during the prenatal months, starting within the first month after conception, when the brain and spinal cord begin to take shape within the embryo. By the sixth prenatal month, nearly all of the billions of neurons (nerve cells) that populate the mature brain have been created, with new neurons generated at an average rate of more than 250,000 per min. Neuronal proliferation and migration are completed well before birth. The outgrowth of axons and dendrites, myelination, synapse formation, and production of neurotransmitters also begins before birth and continues during the first 2 years of life. Once formed, neurons quickly migrate to different parts of the brain and become differentiated to assume specialized roles, so that at birth, the majority of neurons are appropriately located within the immature brain. Neurons form connections (synapses) with other neurons, enabling them to communicate and store information. These synapses continue to form throughout childhood. Axon outgrowth is followed by myelination. Some tracts are myelinated very early (i.e., antenatally), such as the vestibular tracts and some of the motor tracts; others are myelinated much later (e.g., the optical nerves, the auditory system, the cerebellar tracts, and the prefrontal cortex).

The timetable for brain development varies by region and continues throughout life. Sensory regions, which govern sight, touch, hearing, and other sensations, undergo their most rapid growth early in life, whereas the brain areas, such as the prefrontal cortex, which guide higher forms of cognitive thinking and reasoning, continue to experience blooming and pruning of brain connections into early adolescence (14). The prefrontal cortex is one of the last regions of the central nervous system to undergo full myelination, and developmental changes originating from frontal lobe development are evident in several periods of life (14). The most active periods of development of the prefrontal cortex appear to be in the first 2 years of life, then between 7 and 9 years, and finally in adolescence.

The development of the frontal lobe in infants deserves particular mention because during this period, remarkable and rapid changes occur in both the neural physiology and the behavior of the human being. For the frontal cortex, the period of maximum synaptic excess appears to be in the second half of the first year (15), after which, there occurs a protracted period of decline in synaptic density through selective elimination of little used pathways. Studies of human brain activity using positron electron tomography (PET) documented developmental changes in rates of glucose metabolism (16). These changes are characterized by a rise in metabolism in the frontal region at approximately $6-8$ months of age, followed by a prolonged period of decline in rates of metabolism, which parallels the decline in synaptic density (16). Thus, it seems that the significant and rapid changes related to anatomy and function of the frontal lobe occur in the second half of the first year after birth and continue more slowly after this early period.

There is ample evidence that EF also develops dramatically during the second half of the first year of life. For example, infants can hold information in mind for increasing periods and use this information to direct and regulate their responses. They begin to inhibit prepotent responses (17) and become planful, demonstrating the ability to carry out relatively complex sequences of novel behavior (17). Behaviors of this type have been shown in numerous studies to be related to various areas of the prefrontal cortex.

\section{The relation between EF and the prefrontal cortex in human infants}

Diamond and Doar (18) were the first to combine developmental psychology approaches with those of neuropsychology and comparative psychology to assess EF in both animal and human infants using the classic DR task and the $\mathrm{A} B$ task. The DR task procedure was originally used almost exclusively with animals. It requires the subject to watch the experimenter hide an attractive object in one of two identical wells. After a brief delay (the duration of which can be varied), the subject is allowed to search for the object. This sequence is repeated on subsequent trials with the hiding location changed randomly between the two wells.

The standard $A B$ task was originally described by Piaget (19) to measure the changes in the concept of object permanence in human infants. In Piaget's $A B$ task, an infant sits before two identical hiding places, often referred to as 
occluders (e.g., two identical cloth covers or two identical lids) that are separated by a small distance. While the infant watches, a desired object is hidden in one location (A). After a delay, the infant is allowed to reach and search for the object. This hiding and search at location A is repeated. Then, while the infant watches, the object is hidden at the second location (B). After the delay, the infant is allowed to reach and search for the object. Infants frequently make the error of searching again at location $\mathrm{A}$, committing what is known as the classic $\mathrm{A} B$ error. The $\mathrm{A} B$ task has undergone some minor modifications over time so that it allows the effects of time delay on search behavior in human infants to be examined (10). It has many similarities to the DR task but varies in terms of the rules governing when the hiding place is changed.

The performance on DR tasks is believed to be governed by the prefrontal cortex (20). Studies using the DR task with infant monkeys have shown that they are able to retrieve a hidden object. However, when their frontal lobe is lesioned, they are no longer able to carry out this task successfully (21). Diamond et al. (22) found there were similarities in the pattern of the performance on the DR and $\mathrm{A} B$ tasks in primates. Infant monkeys with lesions of the dorsolateral prefrontal cortex showed errors at delays of $2 \mathrm{~s}$ and random or deteriorated performance at delays of $10 \mathrm{~s}$ on the $\mathrm{A} B$ task, despite their extensive preoperative training and their excellent performance with delays of $12 \mathrm{~s}$ before surgery. No recovery of performance occurred in the weeks following surgery (22). In addition, monkeys with dorsolateral prefrontal cortex lesions had poorer performance on the $A B$ task than monkeys with lesions to the hippocampus formation.

Given the evidence of a link between the DR task and the prefrontal cortex and the similarities in performance on the $\mathrm{DR}$ and $\mathrm{A} B$ task in primates, Diamond (23) suggested that the performance of human infants on the $A B$ task may also be governed by the prefrontal cortex. If this is the case, the prefrontal cortex (which had been thought to be dormant in infancy) must be operating if only at a rudimentary level. Further support for this hypothesis came from a study by Diamond and Doar (18), which showed that human infants and infant monkeys display a similar progressive increase in successful performance on $\mathrm{A} B$ and DR tasks although at somewhat different ages. Human infants begin to carry out these tasks successfully at 7.5-8 months of age and show improvement in performance up to about 12 months of age, whereas infant monkeys begin to carry out the task at 1.5 months and show improvement up to 2.5 months. It was suggested that these advances may be due at least to maturational changes in the dorsolateral prefrontal cortex and proliferation of callosal connections between the supplementary motor areas of the left and right hemisphere (21). Although the dorsolateral prefrontal cortex is not fully mature in human infants of 7-12 months, it appears that at this age, it has reached a level of maturity where it can support some critical cognitive functions.

The measurement of frontal scalp-recorded electroencephalograms (EEG) has given more credence to the relationship between the frontal cortex and performance on the $\mathrm{A} B$ task in human infants. Bell and Fox (24) were able to demonstrate a relationship between individual differences in frontal brain electrical activity, as shown in EEG recordings and performance on the $\mathrm{A} B$ task. Infants at 8 months of age who succeeded on the $A B$ task exhibited greater power values in the frontal EEG during baseline recordings than infants who were unable to do the task $(24,25)$. Additional evidence for the importance of prefrontal cortex maturation for the development of EF abilities has come from studies of children with phenylketonuria (26). Even when treated, this genetically transmitted error of metabolism can have the specific consequence of reducing the levels of the neurotransmitter dopamine in the dorsolateral prefrontal cortex. This results in impaired performance on tasks thought to measure $\mathrm{EF}$, such as the $\mathrm{A} B$ task. Thus, both electrophysiological and behavioral data provide support for the relation between the development of the prefrontal cortex and the emergence of EF in the first year of life.

\section{Preterm birth and prefrontal cortex deficits}

As the prefrontal cortex is one of the last regions of the central nervous system to undergo full myelination and is consequently one of the most immature parts of the brain at birth, any hazardous events may have a particularly detrimental effect on its development. The foundation for later brain development is established in the early years; however, the rapid pace and broad scope of early brain growth means that the immature brain is a vulnerable organ. Fetuses of 22-32 weeks of gestation are now viable if cared for in intensive care nurseries. The brain at this point in development is a thin shell of tissue surrounding the cerebral ventricles. Neuroimaging studies using magnetic resonance imaging technique have shown a significant association between brain volumes and gestational age at birth, suggesting that brain development is associated with the degree of fetal immaturity when the transition from intrauterine to extrauterine life occurs. This transition may profoundly disrupt fetal brain development in preterm infants. However, brain development in preterm infants is imperiled not only by exposure to stressful physiological changes, which it is ill-prepared for, in the transition from intrauterine to extrauterine environment but also by medical complications and maternal distress during and after birth.

During this important maturation process in the first year of life, any damage or disturbance to the development of the prefrontal cortex due to disease, trauma, or conditions associated with perinatal risk factors (i.e., extremely low birthweight, shorter gestation age, and medical complications) may therefore lead to executive dysfunction. Diamond and Goldman-Rakic (27) used animal models to examine whether lesions of the dorsolateral prefrontal cortex would have the same effect on infant monkeys as on adult monkeys. Two of the infant rhesus monkeys were tested longitudinally on the $\mathrm{A} B$ and the DR tasks. They received bilateral lesions of the dorsolateral prefrontal cortex at 5.5 months. They were then tested on the $\mathrm{A} B$ task at 6 months. The findings showed that the infant monkeys that had the prefrontal lesions displayed poorer performance on the $\mathrm{A} B$ task than their age-mates who 
did not have the prefrontal lesions. The lesions produced the same effect in infant monkeys as they did in adult monkeys with prefrontal lesions: they all reached incorrectly when the delay increased to $2-5 \mathrm{~s}$ after the toy changed to the new hiding position.

The prefrontal cortex of infants who are born preterm is even more immature and prone to damage from the multitude of adverse medical complications to which these frail infants may be exposed. Lesions or atypical development of the prefrontal cortex occurring as a consequence of these hazardous events may have a detrimental effect on the development of EF, which may have long-term consequences in terms of learning difficulties at school age. Indeed, it has been found that children born preterm are at an increased risk for neurodevelopmental disorders when they reach school age, and it has been suggested that this may be due to early abnormality in the development of the prefrontal cortex and consequently of executive dysfunction (28).

A range of different brain injuries are associated with premature birth that could affect neuropsychological outcomes. Large fluctuations in blood pressure in immature vessels can lead to hemorrhage in the germinal matrix. This may result in intraventricular hemorrhage and ventricular dilatation. Back pressure venous infarction may then occur, leading to a range of focal white and gray matter injuries, frequently associated with the later development of CP. Fluctuations in brain perfusion may also lead to "watershed injury" in the periventricular white matter [periventricular leukomalacia (PVL)] (29), which too may be associated with $\mathrm{CP}$ in a proportion of cases. PVL is seen in $1 \%-3 \%$ of preterm infants $(30,31)$. Mortality in preterm infants with PVL ranges between $30 \%$ and $60 \%$ (31). Although mortality has decreased during the last years, PVL is still considered a disastrous lesion and is associated with adverse neurodevelopmental outcomes with motor, perceptual, and cognitive deficits $(32,33)$.

\section{$E F$ in preterm infants}

Damage to the prefrontal lobe due to trauma and disease in infancy may have lifelong effects on EF abilities and may cause neurodevelopmental disorders during school years $(4$, $34,35)$. In comparison with adults, childhood frontal lobe lesions produce a more pervasive impairment, interfering with the acquisition of age-appropriate EF skills. Most studies on the relationship between EF development and frontal lobe damage in children are based on older children $(11,36)$. However, a recent case study by Anderson et al. (37) reported that an infant who had right frontal region damage at 3 months showed severe learning difficulties and behavioral problems at school and failure in career development in adulthood despite average intelligence (as measured by traditional intelligence tests at school age and during adulthood). The study found that the impairments largely reflected a failure to develop specific EFs, such as the ability to adapt to new situations, inhibit prepotent responses, and plan a sequence of actions to achieve a designated goal. These findings are consistent with the notion that early damage to prefrontal regions can lead to severe disruption of $\mathrm{EF}$, although not significantly affecting many aspects tapped by standard intelligence tests. They also suggest that the prefrontal cortex may have limited neuronal plasticity, which contributes to executive dysfunction if the damage occurs early. This may be due to disruption to the laying down of the neural architectures, which are viewed as the foundation of cognitive development. A detailed examination of the relationship between prefrontal cortex development and the development of EF in a large sample of human infants has not been possible because of the relative lack of obvious prefrontal lesions in infants and the expense and lack of availability of neural imaging technology.

The development of EF as reflected in working memory, inhibition, and planning is age related. Infants at 7-8 months of age start to display the ability to hold information in mind, inhibit prepotent and irrelevant responses, and execute and plan a course of action. These abilities continue to develop rapidly during childhood and adolescence. Any abnormality of the development of EF during this growth period may result in enduring neurodevelopment disorders. A number of investigators have proposed that neurodevelopmental disorders identified in preterm children are linked to impairments of one or more of the components of $\operatorname{EF}(4,8,38)$.

\section{Deficits of working memory}

Working memory, one of the major components of EF, develops at 8-12 months of age. Likewise, in a study of children at 23 and 66 months of age, the older children were found to have better performance on the delayed alternation task than the younger children (39), indicating continued improvement in working memory. Swanson (40) studied an even older age group (6-76 years of age) and found that performance on working memory tasks improved from 6 to 24 years of age and then gradually started to decline at around the age of 35 years. This suggests that working memory continues to improve throughout childhood and into young adulthood $(40,41)$.

Performance on achievement measures of both reading and mathematics has been found to be associated with measures of both verbal and non-verbal working memory ability (40). Preterm infants aged 8-10 months have been found to have poorer performance than full-term counterparts in working memory as measured by $\mathrm{A} B$ task (42). Similarly, preterm children with learning difficulties and low academic achievement at school age have been found to have visual-spatial working memory and verbal working memory deficits (43). For example, problem solving of mathematical word problems is a complex task that requires numerous cognitive operations, such as comprehension, reasoning, and calculation. Prabhakaran et al. (44) suggested that multiple calculations with intermediate steps (e.g., 981×87) are dependent upon frontal lobe functioning. This may be due to an increase in working memory demands necessary for maintenance and manipulation of intermediate steps while problem solving. Verbal working memory is particularly important for the storage and rehearsal of speech-based verbal information and 
has been found to play a specialized role in reading comprehension (45). Impaired memory for digit span and non-word repetition, which measure verbal working memory, has been associated with learning difficulties in preterm children (43). The sense of past and future, afforded by working memory, gives the individual the capacity to understand the content in learning. However, preterm children with neurodevelopmental disorders have difficulty holding events in working memory (43), and consequently, they are less able to formulate future plans than to respond to present events and less able to generate and select response options.

\section{Deficits of inhibition}

The ability to inhibit a prepotent response is also age related and, like working memory, is first demonstrated at about 7-12 months of age (18), with improvements occurring between 18 and 30 months (46) in the primary school years (6-12 years) and again in young adults (18-29 years) (47). According to Barkley (48), deficient inhibition refers to impulsive acts or acting without thinking about the consequences. He described three components of inhibition, namely, inhibition of a prepotent response, interruption of an ongoing response, and inhibition of interference from external stimuli. It has been found that preterm children with learning difficulties also frequently have attention deficits. In a population-based epidemiological study, it has been found that preterm children had attention deficits (49). Preterm children have been found consistently to have deficits in all three aspects of inhibition $(7,50,51)$. This impairs the ability of children with learning difficulties or attention deficits to engage in other appropriate and more timely activities. Consequently, these children miss out opportunities to learn. Tasks assessing inhibition typically require an inappropriate response to be inhibited and irrelevant stimulus to be ignored. In a number of inhibition tasks, such as the Go/No-Go task (52) and the Stroop Word-Color Test (53), and Walk not Walk task, preterm children showed poorer performance than typically developing children $(54,55)$.

\section{Deficits of planning}

Sun et al. $(17,42)$ found that planning ability similarly emerges in infants at 7-8 months of age, and Chen et al. (56) showed that children at 13 months of age were able to plan and execute the necessary course of action to solve ageappropriate problems and use flexibility in the choice of strategies to solve the problems. Bauer et al. (57) reported that at 18-27 months of age, children had developed the ability to represent the goal state of a problem and to plan, monitor, and execute a course of action necessary to achieve it. The planning tasks used with children under 2 years of age typically require them to remove an obstacle to attain a goal. Older children are usually required to generate a path to a goal. For example, the Tower of Hanoi, which requires children to plan a course of action to reach the goal state, has been used with children from 3 years of age, with marked improvements in performance being observed from the ages of 3 to 12 years, when adult levels of performance are achieved $(41,58)$.
Performance on planning tasks has been found to correlate significantly with arithmetic computation and written composition test scores $(59,60)$. Planning involves the delineation of steps or sequences, both cognitive and behavioral, that the individual will follow during the course of action $(57,61)$. Problems with planning may manifest as difficulty sequencing information in temporal order, which may affect syntax of verbal expression and composition in writing, leading to low school achievement in these areas.

Planning also involves the application of systematic strategies rather than trial-and-error responses in reaching the goal (62). Strategies can be used to guide behavior more efficiently and effectively. Such strategies are often critical to managing complex work or problem-solving tasks, particularly where subsets of strategy-guided behavior must be organized into a larger hierarchy to accomplish tasks or goals. Like patients with frontal lobe injuries who are often found to be deficient in task performances because of poor utilization of strategies (63), preterm children with neurodevelopmental disorders have been found to be deficient in the formulation and application of strategies (64).

Tasks assessing planning typically require a sequence of steps to be planned, executed, monitored, and revised in advance of action. The Tower of London and Tower of Hanoi tasks, which are classic tasks for the assessment of planning, require the planning of a series of moves, which are constrained by the rules of the task, in order to move from the start state to the required end state. Preterm children with learning difficulties have been found to have longer latencies (planning time) before commencing on Tower of London problems (65).

\section{Early assessment of EF}

As early brain insult would have long-term implications for neurobehavioral outcome in preterm born infants and the neurodevelopment disorders are related to the deficits of EF, early identification EF deficits in this population is crucial to inform early intervention and prevention practice. Historically, the assessment of EF in infants was thought impossible. However, recent neuropsychological research has suggested that the $\mathrm{A} B$ task and infant planning tasks (17) provide avenues for research on working memory, inhibition of prepotent responses, inhibition of distraction, and planning, which are considered to be components of EF. Similar to the assessment of EF for adults and older children, the assessment of EF in infants also requires working memory, inhibition, and planning. It has been found that the $A B$ task and planning tasks in infants require these components of $\mathrm{EF}$ $(17,27,61,66)$.

\section{AB tasks}

In addition to being able to mentally represent the object, the following abilities are required for infants to carry out the $\mathrm{A} B$ task successfully: [1] memory of the object's hiding location; [2] inhibition of the prepotent response to the previously 
correct location; and [3] inhibition of interference from external stimuli (23). Hence, completion of this task clearly requires components of $\mathrm{EF}$, and it could therefore be regarded as a suitable means of assessing EF in human infants.

\section{Working memory for the location of a hidden object}

Performance on $\mathrm{A} B$ tasks may reflect the emergence of working memory $(23,67)$. The evidence that strongly supports this is Diamond's (68) finding that performance on the $\mathrm{A} B$ task deteriorated with increasing delay between the time when the object was hidden and the time when the infant was allowed to search. This suggests that the memory of the hidden object becomes progressively weaker when the delay increases. The maximum time delay for correct object location in the $A B$ task has been found to be $2 \mathrm{~s}$ for those aged 7-8 months, $5 \mathrm{~s}$ at 9 months, and $10 \mathrm{~s}$ at 12 months (21). At each age, if the delay is reduced by $2-3 \mathrm{~s}$, infants will reach correctly whether the toy is hidden at the first retrieval position or changed to another location. If the delay is increased by 2-3 s, they err even on location A. At all ages, infants' performances improve if they are allowed to strain toward the correct location during the delay (18). It seems that finding a hidden object in position A mostly requires working memory, and increasing the delay time between hiding and retrieval increases the difficulty of the task for infants.

\section{Inhibition of prepotent responses}

Several researchers $(69,70)$ have suggested that a combination of working memory deficits and inability to inhibit repetition of a previously rewarded response causes $\mathrm{A} B$ errors. Diamond suggested that the $\mathrm{A} B$ task sets up a conflict between the ability to use working memory to guide behavior and a conditioned behavioral tendency to repeat a rewarded response when the object has been hidden at location B. Success at location A strengthens the tendency to reach to it by reinforcing this place response and/or establishing a mental set for this response. The more frequently this response is reinforced, the stronger the response tendency becomes. Infants of 8-12 months make $A B$ errors because they fail to resist the "reinforced habit" to repeat the previously successful responses at location A. This is evident from the study in which transparent occluders A and $\mathrm{B}$ were used, and infants still made $\mathrm{A} B$ errors although less frequently than when the occluders were opaque (71). Thus, the learned habit seems at times to be stronger than even the attraction of the object.

Reaching to a previously rewarded location (location A) requires only working memory, but reaching to a new location (location B) requires both working memory and inhibition of the incorrect prepotent response, making the task more difficult and therefore more prone to errors. It is suggested that as the strength of the prepotent response increases because of repeated reinforcement, the ability to inhibit it must also increase if a correct response is to be made to a new location (72).

\section{Inhibiting distraction from external stimuli}

Interference control, or resistance to distraction, is defined by Barkley (49, p. 74) as the ability to "inhibit responding to sources of interference while engaged in another task requiring self-control or while delaying a response". Whether or not distracters disrupt task performance depends upon the strength of the distracter and the potency of the response likely to be elicited by it (48). In the $A B$ task, when a delay time is imposed between hiding and retrieval, external stimuli, such as the cover that is used to hide the object, may act as a distracter that interferes with the performance of the task. Few studies have, however, examined infants' ability to inhibit attention to distracter stimuli.

In summary, the analysis of performance on the $A B$ task shows the importance of EF in completing this task. There is agreement that the EF abilities required to successfully carry out the $\mathrm{A} B$ task are working memory and inhibition of the prepotent response, and consequently, most studies have focused on these factors $(68,69)$. It seems that inhibition to distraction from external stimuli is also required to conduct the $\mathrm{A} B$ task, and few studies have examined this aspect of inhibition.

\section{Planning task}

The majority of research on planning by infants is based on their ability to negotiate means-ends problems that require overcoming obstacles to reach observable goals. For example, Sun et al. $(17,42)$ used a one-step planning task in which infants were presented with a desired toy that was just out of their reach on the far end of a cloth. In order to reach the goal (toy), the infants had to pull the cloth. Infants younger than 6 months of age showed behavior that was neither intentional nor goal directed. For example, they often ignored the goal object or appeared surprised when they eventually noticed it (61).

At 7-8 months, infants were able to sequence the necessary steps to get the toy showing planned, goal-directed action. By 10 months, infants were found to be able to solve more complex problems involving a sequence of two subgoals. For example, a two-step planning task used by Willatts and Rosie (73) required the infants to remove a barrier to gain access to a cloth and then pull the cloth to bring the toy within reach. Infants at 10 months had the capacity to conceptualize the goal of retrieving the toy and could achieve the subgoals of removing the barrier and pulling the cloth to bring the toy within reach so that it could be grasped. At 12 months, they could extend and chain simple means-ends skills in a threestep problem-solving task that required the removal of a barrier to gain access to a cloth, pulling the cloth to retrieve a string, and then pulling the string to bring the toy within reach (74). Infants show steady improvements in their ability to order their actions and to make either reaching or crawling detours when faced with barriers, so that they show flexibility in sequencing their actions and are able to create longer chains of temporally sequenced actions in order to obtain a desired goal $(75,76)$. 
In a cross-sectional study, Chen et al. (56) compared groups of infants at 10 and 13 months on a more complex planning task. The task that they used was somewhat different from that used by Willatts and Rosie (74). In this task, a barrier was put in front of two pieces of cloth, each of which had a string on it. A toy was attached to one string but not to the other. The infant needed to remove the barrier, pull the relevant cloth, and then pull the string to bring the toy within reach. The solution to the problem required choosing the correct subgoal, such as selecting the relevant cloth in order to retrieve the toy. As suggested by Willatts (77), the planning task is more difficult when the infant must initially choose between two subgoals, which determine the achievement of later ones. For example, if the infant chooses the irrelevant cloth (i.e., the one with a string that is not attached to the toy), then no further action will be possible and the problem will remain unsolved. However, if the correct subgoal (i.e., correct cloth) is selected, then the infant can proceed to the next subgoal and will solve the problem. The ability to order a preferred sequence of actions in anticipation of goal conflict necessarily involves planning, which includes mentally representing the effects of first attempting to achieve each subgoal, noticing the conflict of two identical subgoals, (such as two cloths), and finding the solution in advance of any action and without feedback. Findings in the study by Chen et al. (56) showed that infants at both 10 and 13 months used a more or less trial-and-error approach and did not show any planned and intentional actions for the first trial because they could not detect the critical subgoal conflict. However, the performance of the 13-month infant group improved after modeling a solution, suggesting they recognized and understood the conflict even though they failed to plan how to avoid it. Willatts further suggested that the acquisition of this complex meansend strategy starts to develop after 12 months and continues well beyond the period of infancy. Cognitive tasks involving meansend problem solving, which is clearly planned intentionally with a goal in mind, have enabled the planning components of EF to be examined in infants.

Because planning tasks involve a number of steps, there are opportunities for distracters to intervene and disrupt performance. Whether or not this occurs is likely to depend upon the power of the distracter, the level of prepotency of the response elicited by the distracting object or event, and conflict between the subgoal and the goal $(48,61)$. In the onestep planning task, Willatts (61) found that although 6-monthold infants were initially fixated on the toy, they often looked away, got fixated on the cloth, picked it up, and played with it. Thus, the immediate or closest stimulus, which is the cloth, acted as a distracter that interfered with the performance of the planning task. However, by 7-8 months, infants planned in advance and inhibited distractions in order to sequence the steps and achieve the goal, such as retrieval of the toy. Clearly, completion of a planning task requires not only planning but also inhibition of distraction from external stimuli.

\section{Conclusions}

In summary, EF develops rapidly in childhood, with the components of working memory, inhibition, and planning emerging in infancy and improving steadily in the second half of the first year of life. Developmental problems with EF have been shown to underlie neurodevelopmental disorders including learning difficulties and attention deficits in children, and these problems are particularly common in children born preterm. Early assessment of these problems is critical to successful interventions to promote normal development of EF. The use of $A B$ and planning tasks that require components of working memory, inhibition, and planning make early identification of EF deficits in this population possible and can therefore inform early intervention and prevention practice.

\section{References}

1. Blondel B, Macfarlane A, Gissler M, Breart G, Zeitlin J, et al. Preterm birth and multiple pregnancy in European countries participating in the PERISTAT project. Br J Obstet Gynaecol 2006;113:528-35.

2. Michikata K, Sameshima H, Sumiyoshi K, Kodama Y, Kaneko $\mathrm{M}$, et al. Developmental changes in catecholamine requirement, volume load and corticosteroid supplementation in premature infants born at 22 to 28 weeks of gestation. Early Hum Dev 2010;86:401-5.

3. Ferrara TB, Hoekstra RE, Couser RJ, Gaziano EP, Calvin SE, et al. Survival and follow-up of infants born 23 to 26 weeks of gestational age: effects of surfactant therapy. J Pediatr 1994;124:119-24.

4. Arpino C, Compagnone E, Montanaro ML, Cacciatore D, Luca $\mathrm{AD}$, et al. Preterm birth and neurodevelopmental outcome: a review. Childs Nerv Syst 2010;26:1139-49.

5. McQuillen PS, Sheldon RA, Shatz CJ, Ferriero DA. Selective vulnerability of subplate neurons after early neonatal hypoxiaischemia. J Neurosci 2003;23:3308-15.

6. Adams-Chapman I. Neurodevelopmental outcome of the late preterm infant. Clin Perinatol 2006;33:947-64.

7. Marlow N, Hennessy EM, Bracewell MA, Wolke D. Motor and executive function at 6 years of age after extremely preterm birth. Pediatrics 2007;120:793-804.

8. Mulder H, Pitchford NJ, Marlow N. Processing speed and working memory underlie academic attainment in very preterm children. Arch Dis Child Fetal Neonatal Ed 2010;95:F267-72.

9. Huizinga M, Dolan CV, van der Molen MW. Age-related change in executive function: developmental trends and a latent variable analysis. Neuropsychologia 2006;44:2017-36.

10. Miyake A, Friedman NP, Emerson MJ, Witzki AH, Howerter A. The unity and diversity of executive functions and their contributions to complex "Frontal Lobe" tasks: a latent variable analysis. Cogn Psychol 2000;41:49-100.

11. Eslinger PJ, Biddle K, Pennington B, Page RB. Cognitive and behavioral development up to 4 years after early right frontal lobe lesion. Dev Neuropsychol 1999;15:157-91.

12. Aanoudse-Moens CSH, Weisglas-Kuperus N, van Goudoever $\mathrm{JB}$, Oosterlaan J. Meta-analysis of neurobehavioral outcomes in very preterm and/or very low birth weight children. Pediatrics 2009;124:717-28.

13. Volpe JJ. Brain injury in premature infants: a complex amalgam of destructive and developmental disturbances. Lancet Neurol 2009;8:110-24.

14. Gogtay N, Giedd JN, Lusk L, Hayashi KM, Greenstein D, et al. Dynamic mapping of human cortical development during 
childhood through early adulthood. Proc Natl Acad Sci USA 2004;101:8174-9.

15. Huttenlocher PR. Synaptogenesis in human cerebral cortex. In: Dawson G, Fischer KW, editors. Human behavior and the developing brain. New York: The Guilford Press, 1994:137-52.

16. Chugani HT, Phelps ME. Imaging human brain development with positron emission tomography. J Nucl Med 1990;32:23-5.

17. Sun J, Mohay H, O'Callaghan M. Executive function in preterm and full-term infants. Early Hum Dev 2009;85:225-30.

18. Diamond A, Doar B. The performance of human infants on a measure of frontal cortex function, the delayed response task. Dev Psychobiol 1989;22:271-94.

19. Piaget J. The construction of reality in the child. New York: Basic Books, 1954:203-4.

20. Diamond A, Kirkham N, Amso D. Conditions under which young children can hold two rules in mind and inhibit a prepotent response. Dev Psychol 2002;38:352-62.

21. Diamond A. Neuropsychological insights into the meaning of object concept development. In: Johnson MH, editor. Brain development and cognition: a reader. Cambridge, MA: Blackwell, 1993:208-47.

22. Diamond A, Zola-Morgan S, Squire LR. Successful performance by monkeys with lesions of the hippocampal formation on $\mathrm{A} B$ and object retrieval, two tasks that mark developmental changes in human infants. Behav Neurosci 1989;103:526-37.

23. Diamond A. The development and neural bases of memory functions as indexed by the $\mathrm{A} B$ and delayed response tasks in human infants and infant monkeys. The development and neural bases of higher cognitive functions: annals of the New York academy of sciences. New York: The New York Academy of Sciences, 1990:394-433.

24. Bell MA, Fox NA. The relations between frontal brain electrical activity and cognitive development during infancy. Child Dev 1992;63:1142-63.

25. Bell MA. Frontal lobe function during infancy: implications for the development of cognition and attention. In: Richards JE, editor. Cognitive neuroscience of attention: a developmental perspective. Mahwah, NJ: Lawrence Erlbaum, 1998: 287-316.

26. Diamond A, Prevor MB, Callender G, Druin DP. Prefrontal cortex cognitive deficits in children treated early and continuously for PKU. Monogr Soc Res Child Dev 1997;62:1-205.

27. Diamond A, Goldman-Rakic PS. Comparative development of human infants and infant rhesus monkeys of cognitive functions that depend on the prefrontal cortex. Neuropsychol Abstr 1986;12:274.

28. Lowe J, Duvall SW, MacLean PC, Caprihan A, Ohls R, et al. Comparison of structural magnetic resonance imaging and development in toddlers born very low birthweight and full term. J Child Neurol 2011;26:586-92.

29. Rennie JM. The immature brain. In: Rennie JM, editor. Neonatal cerebral ultrasound. Cambridge: Cambridge University Press, 1997:124.

30. Hamrick SEG, Miller SP, Leonard C, Glidden DV, Ramaswamy $\mathrm{V}$, et al. Trends in severe brain injury and neurodevelopmental outcome in premature newborn infants: the role of cystic periventricular leukomalacia. J Pediatr 2004;145:593-9.

31. Roze E, Kerstjens JM, Ter Horst HJ, Maathuis CGB, Bos AF. Risk factors for adverse outcome in preterm infants with periventricular hemorrhagic infarction. Pediatrics 2008;122:46-52.

32. Bassan H, Limperopoulos C, Visconti K, Mayer DL, Feldman HA, et al. Neurodevelopmental outcome in survivors of periventricular hemorrhagic infarction. Pediatrics 2007;120:785-92.
33. Brouwer A, Groenendaal F, van Haastert IL, Rademaker K, Hanlo P, et al. Neurodevelopmental outcome of preterm infants with severe intraventricular hemorrhage and therapy for posthemorrhagic ventricular dilatation. J Pediatr 2008;152:648-54.

34. Eslinger PJ, Grattan LM, Damasio H, Damasio AR. Developmental consequences of childhood frontal lobe damage. Arch Neurol 1992;49:764-9.

35. Scheibel RS, Levin HS. Frontal lobe dysfunction following closed head injury in children: findings from neuropsychology and brain imaging. In: Krasnegor NA, Lyon GR, Goldman-Rakic PS, editors. Development of the prefrontal cortex: evolution, neurobiology, and behavior. Baltimore: Paul H. Brookes, 1997: 241-63.

36. Mateer CA, Williams D. Effects of frontal lobe injury in childhood. Dev Neuropsychol 1991;7:359-76.

37. Anderson SW, Damasio H, Tranel D, Damasio AR. Long-term sequelae of prefrontal cortex damage acquired in early childhood. Dev Neuropsychol 2000;18:281-90.

38. Mulder H, Pitchford NJ, Hagger MS, Marlow N. Development of executive function and attention in preterm children: a systematic review. Dev Neuropsychol 2009;34:393-421.

39. Espy KA, Kaufmann PM, McDiarmid MD, Glisky ML. Executive functioning in preschool children: performance on A-not-B and other delayed response format tasks. Brain Cogn 1999;41:178-99.

40. Swanson HL. What develops in working memory? A life span perspective. Dev Psychol 1999;35:986-1000.

41. Welsh MC, Pennington BF, Groisser DB. A normativedevelopmental study of executive function: a window on prefrontal function in children. Dev Neuropsychol 1991;7:131-49.

42. Sun J, Buys N. Prefrontal lobe functioning and its relationship to working memory in preterm infants. Int J Child Adolesc Health 2011;4:13-7.

43. Gathercole SE, Pickering SJ. Working memory deficits in children with low achievements in the national curriculum at 7 years of age. Br J Educ Psychol 2000;70:177-94.

44. Prabhakaran V, Rypma B, Gabrieli JDE. Neural substrates of mathematical reasoning: a functional magnetic resonance imaging study of neocortical activation during performance of the necessary arithmetic operations tests. Neuropsychology 2001;15:115-27.

45. Nation K, Adams JW, Bowyer-Crane CA, Snowling MJ. Working memory deficits in poor comprehenders reflect underlying language impairments. J Exp Child Psychol 1999;73: 139-58.

46. Vaughn BE, Kopp CB, Krakow JB. The emergence and consolidation of self-control from eighteen to thirty months of age: normative trends and individual differences. Child Dev 1984;55:990-1004.

47. Williams BR, Ponesse JS, Schachar RJ, Logan GD, Tannock R. Development of inhibitory control across the life span. Dev Psychol 1999;35:205-13.

48. Barkley RA. Behavioral inhibition, sustained attention, and executive functions: constructing a unifying theory of ADHD. Psychol Bull 1997;121:65-94.

49. Harvey JM, O'Callaghan MJ, Mohay H. Executive function of children with extremely low birthweight: a case control study. Dev Med Child Neurol 1999;41:292-7.

50. Bohm B, Katz-Salamon M, Smedler A, Lagercrantz H, Forssberg H. Developmental risks and protective factors for influencing cognitive outcome at $51 / 2$ years of age in verylow-birthweight children. Dev Med Child Neurol 2002;44: 508-16. 
51. Katz KS, Dubowitz LM, Henderson S, Jongmans M, Kay GG, et al. Effect of cerebral lesions on continuous performance test responses of school age children born prematurely. J Pediatr Psychol 1996;21:841-55.

52. Schachar R, Mota VL, Logan GD, Tannock R, Klim P. Confirmation of an inhibitory control deficit in attention-deficit/ hyperactivity disorder. J Abnorm Child Psychol 2000;28: 227-35.

53. Pennington BF, Groisser D, Welsh MC. Contrasting cognitive deficits in attention deficit hyperactivity disorder versus reading disability. Dev Psychol 1993;29:511-23.

54. Atkinson J, Braddick O. Visual and visuocognitive development in children born very prematurely. Prog Brain Res 2007;164:123-49.

55. Bayless S, Stevenson J. Executive functions in school-age children born very prematurely. Early Hum Dev 2007;84: 247-54.

56. Chen Z, Sanchez RP, Campbell T. From beyond to within their grasp: the rudiments of analogical problem solving in 10- and 13-month-olds. Dev Psychol 1997;33:790-801.

57. Bauer PJ, Schwade JA, Wewerka SS, Delaney K. Planning ahead: goal-directed problem solving by 2-year-olds. Dev Psychol 1999;35:1321-37.

58. Passler MA, Isaac W, Hynd GW. Neuropsychological development of behavior attributed to frontal lobe functioning in children. Dev Neuropsychol 1985;1:349-70.

59. Hooper SR, Swartz CW, Wakely MB, de Kruif REL, Montgomery JW. Executive functions in elementary school children with and without problems in written expression. J Learn Disabil 2002;35:57-68

60. Snow JH. Developmental patterns and use of the Wisconsin Card Sorting Test for children and adolescents with learning disabilities. Child Neuropsychol 1998;4:89-97.

61. Willatts P. Development of means-end behavior in young infants: pulling a support to retrieve a distant object. Dev Psychol 1999;35:651-67.

62. Scholnick EK, Friedman SL. Planning in context: developmental and situational considerations. Int $\mathrm{J}$ Behav Dev 1993;16:145-67.

63. Stuss DT, Eskes GA, Foster JK. Experimental neuropsychological studies of frontal lobe functions. In: Boller F, Spinnler H, Hendler JA, editors. Handbook of neuropsychology. Amsterdam: Elsevier, 1994:149-85.
64. Keeler ML, Swanson HL. Does strategy knowledge influence working memory in children with mathematical disabilities? J Learn Disabil 2001;34:418-34.

65. Luciana M, Lindeke L, Georgieff M, Mills M, Nelson CA. Neurobehavioral evidence for working-memory deficits in school-aged children with histories of prematurity. Dev Med Child Neurol 1999;41:521-33.

66. Diamond A. Frontal lobe involvement in cognitive changes during the first year of life. In: Gibson KR, Peterson AC, editors. Brain maturation and cognitive development: comparative and cross-cultural perspectives foundations of human behavior. New York: Aldine De Gruyter, 1991:127-80.

67. Gilmore RO, Johnson MH. Working memory in infancy: sixmonth-olds' performance on two versions of the oculomotor delayed response task. J Exp Child Psychol 1995;59:397-418.

68. Diamond A. Development of the ability to use recall to guide action, as indicated by infants' performance on $\mathrm{A} B$. Child Dev 1985;56:868-83.

69. Diamond A. Abilities and neural mechanisms underlying A $B$ performance. Child Dev 1988;59:523-7.

70. McCall DD, Clifton RK. Infants' means-end search for hidden objects in the absence of visual feedback. Infant Behav Dev 1999;22:179-95.

71. Butterworth G. Object disappearance and error in Piaget's stage IV task. J Exp Child Psychol 1977;23:391-401.

72. Diamond A, Werker JF, Lalonde C. Toward understanding commonalities in the development of object search, detour navigation, categorization, and speech perception. In: Dawson G, Fischer KW, editors. Human behavior and the developing brain. New York: The Guilford Press, 1994:380-426.

73. Willatts P, Rosie K. Thinking ahead: development of means-end planning in young infants. Infant Behav Dev 1992;15:769.

74. Willatts P, Rosie K, editors. Planning by 12-month-old infants. Annual Conference of the Society for Research in Child Development, Kansas City, MO, April 1989.

75. Lockman JJ, Pick HL. Problems of scale in spatial development. In: Sophian C, editor. Origins of cognitive skills. Hillsdale, NJ: Lawrence Erlbaum, 1984:3-26.

76. McKenzie BE, Bigelow E. Detour behavior in young human infants. Br J Dev Psychol 1986;4:139-48.

77. Willatts P, editor. Development of means-end planning in the second year of life. International Conference on Infant Studies, Atlanta, GA, April 1998. 